\title{
IoT Enabled, Leaf Wetness Sensor on the Flexible Substrates for In-situ Plant Disease Management
}

\author{
Kamlesh S. Patle, Riya Saini, Ahlad Kumar, Sandeep G. Surya Member, IEEE, Vinay S Palaparthy, \\ Khaled. N. Salama Senior Member, IEEE
}

\begin{abstract}
Early plant disease detection and providing the control measures have become highly desirable to improve crop yield. Leaf wetness duration (LWD) is one of the essential parameters related to the development of fungal disease on the leaf canopy. To measured LWD, the leaf wetness sensor (LWS) is widely used. Commercially available LWS are made on printed circuit board (PCB) technology, which has an operational issue during field deployment such as weight of the sensor, contact resistance between the sensor and the leaves, form factor and most importantly, affordability. To mitigate the issues associated with the commercially available LWS, in this work, we have fabricated the in-house loT-enabled and affordable electronic leaf wetness sensor on the flexible substrates, which is used for integrated plant disease management. Fabricated LWS comprises the interdigitated electrodes (IDEs) on the polyimide flexible substrate. The lab measurement results indicate that fabricated LWS on the flexible substrates offers a response of about $36000 \%$ when LWS is exposed to water w.r.t air. The observed response time of the fabricated LWS is about 10 seconds and hysteresis of about $\pm 4 \%$. Further, sensor capacitance changes only by $6 \%$ over a temperature range from $20^{\circ} \mathrm{C}$ to 65 ${ }^{\circ} \mathrm{C}$. Furthermore, three fabricated sensors LWS and in-house developed internet of things (IOT) enabled systems are deployed on the Ocimum tenuiflorum (Tulsi) medical plant. Field measurement indicates that measured LWD using the fabricated flexible LWS and commercially available LWS (Phytos 31:LWS-L12), METER Group, Inc. USA) shows the absolute difference of $\mathbf{3 0}$ minutes.
\end{abstract}

Index Terms_-Leaf wetness sensor, flexible substrates, plant disease management, agriculture applications

\section{INTRODUCTION}

he plant diseases, weeds, and pests in the agricultural

field lead to almost $36 \%$ loss in crops [1]. This decreases the crop yield and can lead to grave circumstances for the farmers of low-income countries. The rationale behind the crop loss is attributed to the irregularly followed agriculture rehearses, late recognition of the plant disease due to the weeds and pests. To mitigate such loss, sensor-based technology is pertinent and plays a vital role in the early diagnosis of plant diseases. The new progression in sensor development has opened up an avenue to improve crop yield and reduce the effect crops losses. To alleviate the crop loss due to plant diseases, researchers across the globe have proposed early plant disease detection models wherein leaf wetness duration (LWD), ambient temperature, and relative humidity are few significant parameters required during in-situ testing [2-4].

The authors are thankful the Department of Science and Technology - Science and Engineering Research Board (DSTSERB) for financial assistance received through a start-up research grant (SRG) FILE NO. SRG/2019/000895.
The presence of water particles (acting as free water) on plant leaves, along with appropriate environmental conditions such as ambient temperature and humidity, leads to the germination of fungal diseases on the leaves [5]. The sprinklers or rain or dew are the primary source of water droplets on the leaf canopy. The leaf wetness sensor (LWS) is generally employed to quantify the LWD [6]. The reported LWS is categorized as static, mechanical, and

\footnotetext{
${ }^{*}$ Kamlesh S. Patle is with System Design Lab, Department of Information and Communication Technology, DAIICT, Gandhinagar, 382007, Guajrat, India.

${ }^{*}$ Riya Saini is with System Design Lab, Department of Information and Communication Technology, DAIICT, Gandhinagar, 382007, Guajrat, India.

Ahlad Kumar is with System Design Lab, Department of Information and Communication Technology, DAlICT, Gandhinagar, 382007, Guajrat, India.

Sandeep G. Surya was with Sensors Lab, Advanced Membranes \& Porous Materials Center (AMPMC), CEMSE, King Abdullah University of Science and Technology (KAUST), Thuwal, 23955-6900, Saudi Arabia

Vinay S Palaparthy is with System Design Lab, Department of Information and Communication Technology, DAlICT, Gandhinagar, 382007, Guajrat, India.

K. N. Salama is with Sensors Lab, King Abdullah University of Science and Technology (KAUST), Thuwal, 23955-6900, Saudi Arabia

${ }^{*}$ Authors have equal contributions
} 
electronic [7-9]. The static LWS provides the information on whether the leaf canopy is dry or wet; the mechanical LWS notices the change in sensor weight or length with the presence of wetness on the leaf. At the same time, the electronic LWS observes the change in sensor capacitance or resistance due to the presence of wetness on the leaves. The advantage of the static and mechanical LWS is that they are easy to use. However, the primary concern for the static and mechanical LWS is stability and reliability under in-situ conditions [7-9]. On the other hand, electronic LWS offers more stability and reliability, but it is not affordable to the framers. Among the resistive and capacitive electronic LWS, capacitive LWS is widely used due to its precision and stability for in-situ applications.

Researchers have widely explored the electronic LWS over static and mechanical LWS as one of the potential contenders for the laboratory and as well as for the field insitu LWD measurements, considering its stability, accuracy, and precision. The copious testing of the electronic LWS for in-situ LWD measurements is reported by different researchers [10-21]. The electronic LWS is placed as the artificial leaves near the leaf canopy. These sensors are fabricated either on the printed circuit board (PCB) or on a ceramic plate, which has an interdigitated electrode (IDE) as the sensing electrodes [23-24]. Even the commercially available leaf wetness sensors use the sensing electrodes mounted on the printed circuit board PCB [21]. There are, however, a few issues associated with the PCB-based leaf wetness sensors. In the PCB sensor, there is an issue of the contact resistance between the sensor and leaf, resulting in inaccurate measurements during the in-situ measurements. PCB-based sensors are comparatively heavy, which bends the leaf during the deployment. Further, scientists have investigated the in-situ LWS performance considering the operational aspect of the sensors [10-19]. From various investigations, it was observed that in-situ LWD measurements are problematic due to a lack of standard establishment and operational aspects. They include sensor orientation and placement, weight and size, and the effect of diurnal temperature variations [25-27]. The issues mentioned above associated with the operational exposure of the sensor hinder the use of LWS for accurate in-situ measurements. Thus, to address the aforementioned issues related to the commercially available and reported leaf wetness sensors, in this work, we propose a new design and development of a state-of-the-art LWS. It is flexible, lightweight, highly sensitive, reliable, less prone to diurnal temperature variations, and, most importantly, affordable.

With the motivation of fabricating the flexible, lightweight temperature-insensitive LWS, in this study, we have fabricated a Yt laser engraved LWS on the flexible polyimide substrate and developed an efficient IoT-enabled and low-power signal conditioning circuit. Considering the operational aspects of the LWS, we have examined the effect of temperature variations on the fabricated flexible LWS. Fabricated LWS comprises aluminum IDEs on the polyimide flexible substrate. In-house developed interface electronics (signal conditioning and processing circuit) measures the change in the fabricated flexible LWS response. In this work, we also established the benchmarking protocol to verify the accuracy of the measured LWD using the fabricated flexible LWS. The benchmark is done in comparison with commercially available and expensive LWS ((Phytos 31: LWS-L12) from METER Environment, METER Group, Inc. USA [21]. For the field measurements, fabricated LWS and system are deployed on the Ocimum tenuiflorum (Tulsi) medical plant. We have also employed the data pre-processing computational methods used for feature extraction to determine LWD using fabricated flexible LWS and benchmark with commercially available LWS Phytos 31 .

\section{MATERIAL AND MetHOdS}

\section{A. Sensor Fabrication on the Flexible Substrates}

Fig. 1 (a) shows the process flow for fabricating the leaf wetness micro-sensors on the flexible substrate. First, the designs of the IDE were made in corel draw software, and it was followed by procurement of polyimide flexible substrate as shown in Fig. 1 (a), then a blank aluminum (Al) deposited is done on the polyimide substrate using the electron beam evaporation method as shown in Fig. 1 (b). Further, the interdigitated electrodes (IDEs) will be patterned using laser ablation (universal laser systems) as shown in Fig. 1 (c), Fig. 1 (d) shows the fabricated IDEs on the flexible substrates. We used $1.06 \mu \mathrm{m} \mathrm{Yb}$ fiber laser to attain the structures and the dimensions of IDEs are made in a way to fit on the leaves of the Ocimum tenuiflorum (Tulsi) medical plant. Further, the system was operated in Rast/Vect mode with other optimized parameters (Power $=20 \%$, speed $=10 \%$, freq $=30 \mathrm{kHz}$ and $\mathrm{Z}$-axis $=2$ $\mathrm{mm})$. Electrical contact from the fabricated sensor is achieved by mounting it on the PCB using silver epoxy, as shown in Fig. 2 (d).

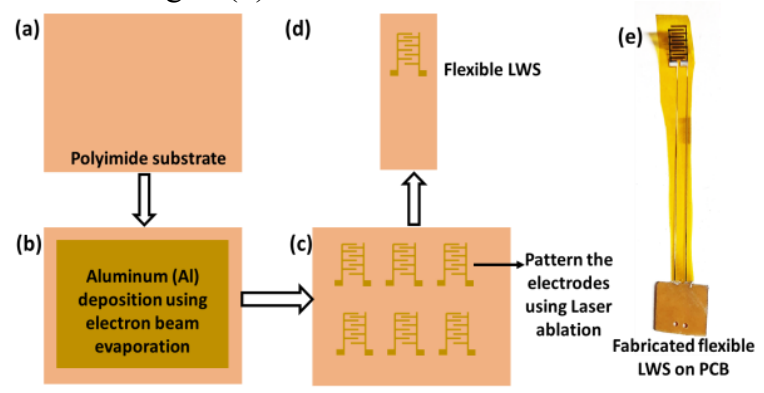

Fig. 1 Fabrication flow for the proposed flexible leaf wetness sensors 

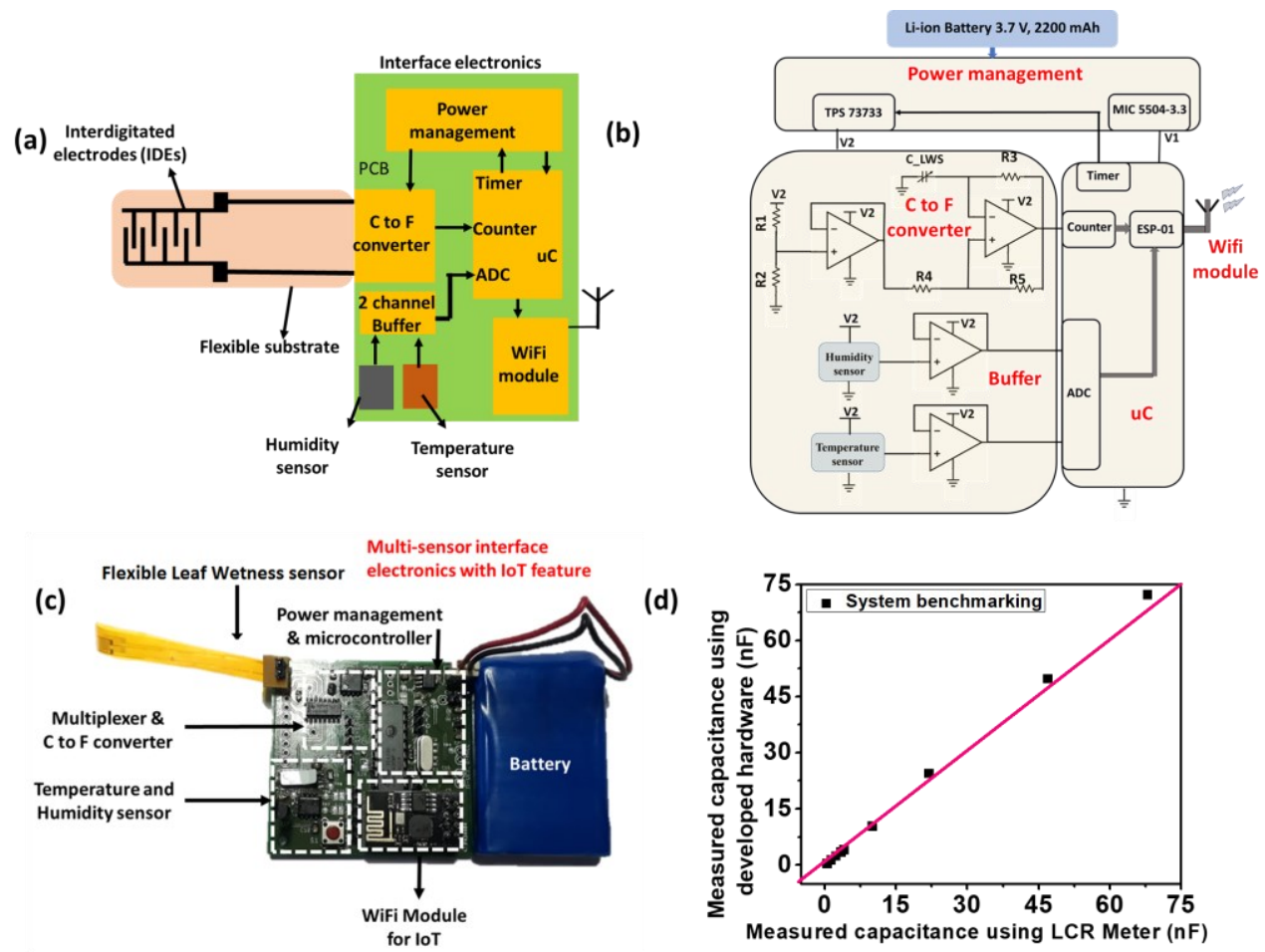

Fig. 2 (a) Schematic representation of the fabricated flexible LWS along with interface electronics, (b) Internal architecture of the interface electronics for the fabricated flexible LWS, (c) Developed hardware for the fabricated sensor embedded with IoT enabled feature, (d) Benchmarking of the measured capacitance from the developed hardware with standard LCR meter

\section{B. Interface electronics for the fabricated flexible LWS}

Fig.2 shows the design and development of the interface electronics for the fabricated flexible LWS wetness sensor along with ambient temperature and humidity sensors and interface electronics and benchmarking. In this work, the capacitive leaf wetness sensor on the flexible substrate is fabricated, which comprises interdigitated electrodes (IDEs) as depicted in Fig. 2 (a). The capacitive LWS operates on the principle of measurement of the dielectric of water (80), which is high when compared with air (1-5) [28]. Hence, the availability of water molecules on the IDEs strengthens the polarization, thereby increasing the dielectric constant, which increases the LWS capacitance [30]. The IDEs are used for this purpose because of the higher surface area for the measurements enhancing sensor sensitivity [31-33]. For plant disease management, in addition to the LWS, the ambient temperature and humidity sensors are also essential to examine the growth of the fungus on the leaf canopy. The interface electronics also comprises of capacitance to frequency converter ( $\mathrm{C}$ to $\mathrm{F}$ ), temperature and humidity sensor, a power management unit, micro-controller, and WiFi module for the internet of things (IoT) communication as shown in Fig. 2 (a).

The detailed internal architecture of the interface electronics developed for the proposed flexible LWS is shown in Fig. 2 (b). It also comprises of relaxation oscillator ( $\mathrm{C}$ to $\mathrm{F}$ converter) that measures the LWS sensor $\left(\mathrm{C}_{\mathrm{LwS}}\right)$ change in the capacitance and convert it to the desired frequency by using (1).

$$
F=\frac{1}{2.2 R C_{-L W S}}
$$

Where $F$ is the measured output frequency of the $\mathrm{C}$ to $\mathrm{F}$ converter, $\mathrm{R}$ is the feedback resistor and C LWS is the fabricated LWS capacitance. Further, in the interface electronics, we have used commercially available HIH 5030 humidity sensor [34] and MCP 9701A temperature sensor [35], whose analog output is given to the ADC of the micro-controller through a unity gain buffer as shown in Fig. 2 (b). Subsequently, the microcontroller $(\mu \mathrm{C})$ ATMEGA328P [36] reads the frequency output of the C to $\mathrm{F}$ converter and analog output of the HIH 5030 \& MCP 9710 using counter and ADC, respectively. Measured data from the fabricated LWS and temperature and humidity sensors are processed and uploaded to the cloud (ThingSpeak ${ }^{\mathrm{TM}}$ ) using a WiFi module (ESP-01) [37] as shown in Fig. 2 (b). The entire system is powered using the 3.7 V and $2200 \mathrm{mAh} \mathrm{Li-ion} \mathrm{batteries.} \mathrm{The} \mu \mathrm{C}$ is powered with a $3.3 \mathrm{~V}$ regulated power supply by using the LDO MIC 5504-3.3 (V2). Whereas the regulated $3.3 \mathrm{~V}$ DC supply to all sensors, C to F converter, buffer, and ESP-01 modules is provided by LDO TPS 73733 (V2), as shown in Fig 2 (b). We have used the power gating scheme for the developed hardware to reduce energy consumption during in-situ operations. To achieve this, we periodically turn on/off the power (V2) delivered to the sensors, $\mathrm{C}$ to $\mathrm{F}$ converter, buffers, and ESP-01 module using the timer of $\mu \mathrm{C}$ for 30 minutes.

Fig. 2 (c) shows the developed hardware for the proposed sensor. It comprises multiple sensors viz. fabricated LWS, humidity, and temperature sensors required for in-situ plant disease management. As shown in Fig. 2 (c), the developed 
hardware is powered with the Li-ion battery. It comprises of WiFi module to send the measured data to the cloud. Further, we have benchmarked the capacitance measured from the developed interface electronics with the commercially available LCR meter (HIOKI IM3536) to ensure the sensor's accuracy [38]. Fig. 2 (d) shows that measured capacitance from the developed interface electronics with commercial LCR meter has an average relative error of about $3 \%$.

\section{Experimental Set-up}

Our study explored the potential use of the fabricated LWS to measured LWD, which is highly correlated with the diseases in plants [11-12]. However, for the LWS, operational aspects and calibration are few significant concerns during in-situ measurements. Thus, in this work, we have attempted to calibrate the fabricated LWS and subsequently validate the calibration by benchmarking with the commercially available LWS (Phytos 31: LWS-L12) from METER Environment, METER Group, Inc. USA. For this purpose, we have divided the sensing area (surface area) of the fabricated flexible LWS as $0 \%, 25 \%, 50 \%$, $75 \%$, and $100 \%$, and water is sprayed on the defined sensing area as shown in Fig. 3. Sensing area wetness is termed as the surface (sensing) area of the fabricated sensor covered with the water molecule. To calculate the sensing area wetness, we have assumed (approximated) the sensor's sensing area as rectangle and then $0 \%, 25 \%, 50 \%, 75 \%$ and $100 \%$ is covered with the water droplets, where $0 \%$ and 100 $\%$ represent the completely dry and wet surface, respectively. Fig. 3 (inset image) shows the mechanism to calculate sensing area wetness. From Fig. 3, it is evident that first, we measured the sensor capacitance with no spray on the sensor, which gives $0 \%$ sensing area wetness. Subsequently, we have sprayed the $25 \%$ area with the water molecule, which is termed as $25 \%$ sensing area wetness. Likewise, we sprayed on the $75 \%$ and $100 \%$ sensing area covered with the water molecules. Fabricated flexible LWS shows the change in the capacitance when exposed to the water, which is measured using the LCR meter (HIOKI IM3536) as depicted in Fig. 3. To avoid the electrolysis of the water molecules, for all the measurements, the excitation voltage of the LCR meter is maintained at $1 \mathrm{Vp}-\mathrm{p}$ [30]. The details of the fabricated flexible LWS transfer characteristics are explained in section II. D.

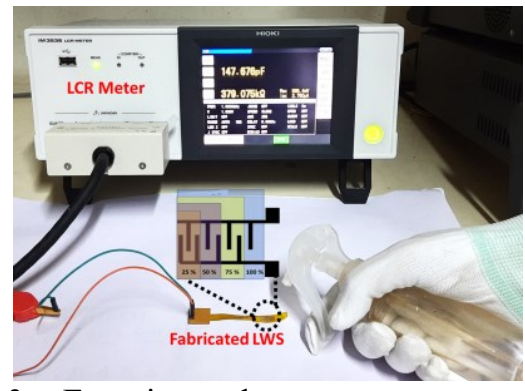

Fig. 3 Experimental setup to generate fabricated LWS transfer characteristics

\section{LWS transfer characteristics}

To understand the fabricated flexible LWS transfer characteristics viz. sensitivity, response time, hysteresis, accuracy, etc., we have first tested the sensor under laboratory conditions. As discussed in the previous section (II.C), to generate the fabricated LWS transfer characteristics and benchmarking, we divided the sensing area (surface area) as $0 \%, 25 \%, 50 \%, 75 \%$, and $100 \%$. Then, water molecules are sprayed on the fabricated flexible LWS, as shown in Fig. 3. During the measurements, $0 \%$ and $100 \%$ surface area wetness are considered dry and wet conditions. First, we have analyzed the sensor's frequency response under dry conditions (no water molecules on the sensor's sensing area), where the fabricated sensor is connected to the LCR meter (as shown in Fig. 3) and the frequency of the LCR meter is varied from $100 \mathrm{~Hz}$ to $3 \mathrm{MHz}$ keeping excitation voltage constant at $1 \mathrm{~V}$. Simultaneously, the capacitance of fabricated LWS is recorded using the LCR meter, as shown in Fig. 4 (a). From Fig. 4 (a), it is evident that LWS capacitance after 10 $\mathrm{kHz}$ becomes constant. Fig 4. (b) shows the change in the LWS capacitance w.r.t the water molecules covering different sensing areas. Fig 4 (b) shows that fabricated LWS capacitance increases monotonically as water molecule covering different sensing areas increases. An increase in the LWS capacitance due to presence of water molecule is attributed to an strengthen in the polarization between IDEs when water molecules on the sensing area increases and thus increase in the dielectric constant is apparent, which leads to rise in the LWS sensor capacitance [29]. Further, we have analyzed the response $(\Delta \mathrm{C} / \mathrm{C})$ of the fabricated flexible LWS, which is calculated using (2) and shown in Fig. 4 (c). From Fig. 4 (b), it is evident that the response of the fabricated LWS is about $36300 \%$ to $35800 \%$ at $500 \mathrm{~Hz}$ and $1 \mathrm{kHz}$, respectively, when the sensing area is completely wet $(100 \%$ sensing area wetness) w.r.t to the dry $(0 \%$ sensing area wetness).

Response $=\left(\frac{\Delta C}{C} \times 100\right) \%=\left(\frac{C_{L W S}-C_{b}}{C_{b}} \times 100\right) \%$

where $C_{L W S}$ is the capacitance of the fabricated LWS when $\mathrm{x} \%$ of the sensing area is wet, and $C_{b}$ is the base capacitance, under dry $(0 \%)$ sensing area conditions. From Fig. 4 (c), it is also evident that sensor response drops as frequency is increased to $10 \mathrm{kHz}$. It is attributed as follows, as the change in the direction of the electric field is high when the frequency is increased. The net polarization of the water molecules on the IDEs surface does not catch up with high frequency. Thus, dielectric constant tends to stable and does not change [29]. Further, we have examined the hysteresis of the fabricated flexible LWS, as shown in Fig. 4 (d). For this purpose, water is sprayed on the sensor, and flexible LWS response is analyzed by increasing the percentage of sensing area covered with the water molecule (i.e., $0 \%$ to $100 \%$ ) (named as adsorption). It is followed by removing the water from the sensing area (i.e., $100 \%$ to $0 \%$ ) (named as desorption), as shown in Fig. 4 (c). Fig. 4 (d) shows that the sensor exhibits a hysteresis of about \pm $5 \%$. 

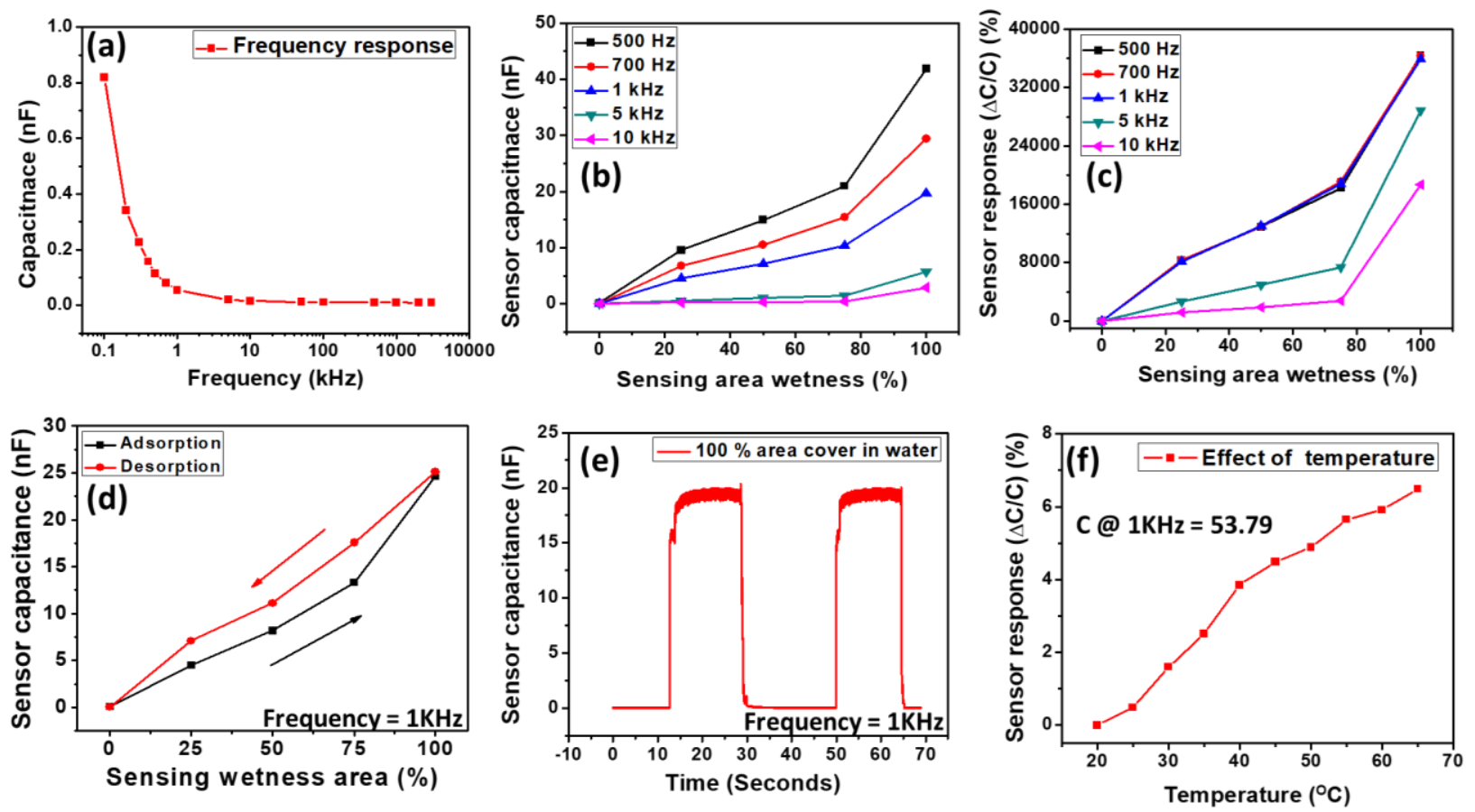

Fig. 4 (a) Change in the sensor capacitance under dry condition for different frequency (b) Change in the fabricated flexible LWS for percentage of sensing area covered with the water molecule (surface area), (c) Response of the fabricated flexible LWS percentage of sensing area covered with the water molecule (d) Hysteresis observed for the fabricated LWS, (e) Response time of the sensor, which is about 10 seconds, (f) Effect of the temperature on the fabricated sensor

Further, we have analysed the response time of the fabricated flexible LWS as shown in Fig. 4 (e). We have fixed the operating frequency at $1 \mathrm{kHz}$ for the response time study considering the sensitivity and repeatability. Then, we have sprayed water on $100 \%$ of the surface area of the fabricated LWS and measured the sensor capacitance as depicted in Fig. 4 (e). From Fig. 4 (e), we can infer that the sensor's response time is about 5 seconds. Furthermore, we have examined the effect of temperature on the flexible LWS, which is one of the primary concerns considering the operational exposure of the LWS. For this purpose, we have deployed the sensor in the hot air oven and varied the temperature from $20{ }^{\circ} \mathrm{C}$ to $65{ }^{\circ} \mathrm{C}$ considering the in-situ agriculture condition in India. Fig. 4 (f) shows the effect of temperature on the flexible LWS, where it is evident that sensor capacitance changes only by $6 \%$ when temperature changes from $20^{\circ} \mathrm{C}$ to $65^{\circ} \mathrm{C}$.

\section{FIELD DEPLOYMENTS}

\section{A. Experimental Set-up for benchmarking}

One of the primary objectives of the field deployments is to benchmark the measured LWD using fabricated flexible LWS with commercial LWS (Phytos 31) from the METER Environment, METER Group, Inc. USA. For this purpose, we have calibrated the Phytos 31 (as shown in Fig. 5 (a)) by using the experimental set-up described in section II. C. Further, water is sprayed on the defined sensing area and the response of Phytos 31 is studied. Fig. 5 (b) shows the response of the Phytos 31 with water molecule covering different sensing areas. The Phytos $31 \mathrm{LWS}$ voltage value increases with an increase in the water molecules coverage on different sensing areas, which is in agreement with [21]. Fig. 5 (c) shows the benchmark experimental set-up to measure LWD using fabricated LWS with Phytos 31 LWS. As shown in Fig. 5 (c), the fabricated flexible LWS output is connected to the LCR meter to measure the change in capacitance and Phytos 31 LWS has been excited with 3.3 V DC voltage, and its output is connected to the digital multimeter. Subsequently, water is sprayed on both the sensor and sensing area. Wetness is measured using both the sensors, as shown in Fig. 5 (d). From Fig. 5 (d), it is evident that the absolute error observed for the fabricated LWS is within $\pm 3 \%$ (sensing area wetness) when benchmarked with the Phytos 31 .
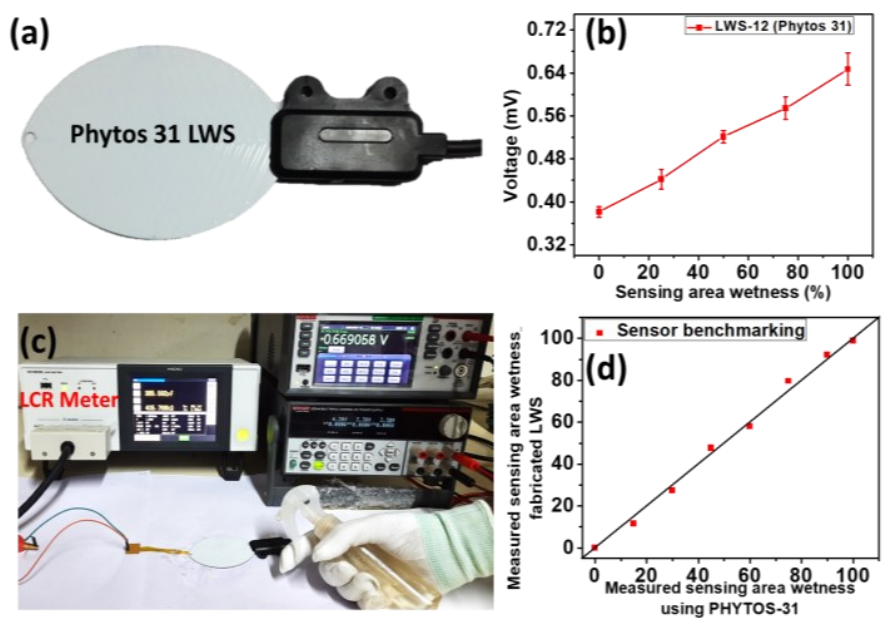

Fig. 5 Experimental setup to benchmark the percentage of sensing area covered with the water molecule measured using fabricated LWS and Phytos 31 


\section{B. Field deployment}

In this study, we have developed a three-sensor network system viz. Node 1 (S1), Node 2 (S2), Node 3 (S3), and deployed close to the Tusli plant. Fig. 6 (a) shows the developed prototype for the in-situ measurements, which comprises fabricated flexible LWS and Phytos 31 LWS (in S1) for benchmarking. During in-situ measurements, both the sensors are deployed at a distance of $30 \mathrm{~cm}$ from the surface and at a 45-degree angle [27] from the surface, as shown in Fig. 6 (a). In this study, the developed sensor, system and commercial LWS are deployed close to the Ocimum tenuiflorum (Tulsi) medical plant leaves. Further, measured data from the fabricated flexible LWS and Phytos 31 LWS are sent to the ThingSpeakTM server using the in-built WiFi on the developed system. The data sampling rate for this study is kept at 30 minutes. During field measurements, a sprinkler is used as a water droplet source on the sensors and on the leaves. The controlled experiments are carried out, where the ON and OFF time of the sprinkler are controlled manually for all the sensors, to ensure that water droplets formed on the leaf and sensors stay for the same amount of time. Section IV describes the measured data recorded from three nodes and the estimation of LWD from the fabricated flexible LWS and Phytos 31 LWS.

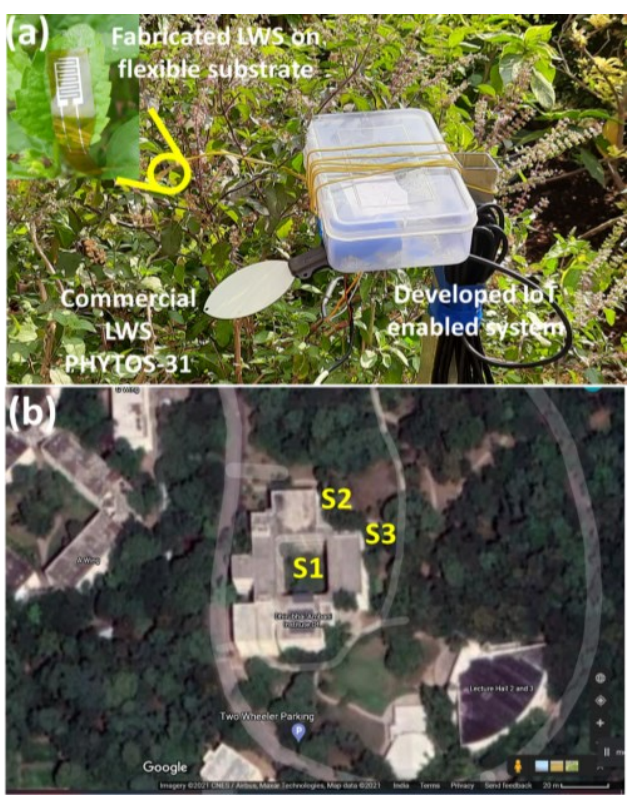

Fig. 6 (a) Developed LWS sensor and system deployed on the Tulsi medical plant (b) location of three nodes deployed for in-situ measurements

\section{DATA ANALYSIS}

\section{A. Data Pre-processing}

Feature extraction is the method of obtaining useful information from a set of data. In this study, the data from the fabricated LWS sensor is the variation of frequency across time due to a change in capacitance of the sensor. Similarly, the resultant data from commercial LWS is changing voltage across time due to the presence of water on the sensor's surface. We can extract Leaf Wetness
Duration (LWD) values from these signals, which will provide us a much better insight into plant disease management. This whole process is an instance of feature extraction.

This study captured more than 1600 LWD values over one month at an interval of 30 minutes, as shown in Fig. 7. Fig. 7 depicts the collected raw data from three different nodes deployed in the field viz. Node 1 (S1), Node 2 (S2), Node 3 (S3) and 1 node with commercial LWS (PHYTOS 31). Before trying to estimate the LWD values, we must apply some data cleaning and processing techniques to improve our data quality $[39,40]$. This process is called data pre-processing. The data collected from the sensors vary greatly in terms of the range of values. To get a better insight and introduce uniformity, we perform a scaling operation on all of them. For this purpose, we have used a scaling technique called normalization. We used MinMax Scaler [41], which is a widely used scaling algorithm on different data. We converted the range of values of the signal obtained from the sensors from 0 to 1 . Fig 7 represents all the signals recorded from the four sensors (three flexible LWS and one Phytos 31 LWS). All the signals have data that varies between 0 and 1. Signals from fabricated sensors have been mirrored with respect to y-axis so as to have a baseline with lowest values for all sensors. The normalization function is readily available in the scikitlearn library, for use in Python. The equation used for the transformation is as follows [41]:

$X_{\text {std }}=(X-X \cdot \min ($ axis $=0)) /(X \cdot \max ($ axis $=0)-X \cdot \min ($ axis $=0))$

$$
X_{\text {scaled }}=X_{\text {std }} *(\max -\min )+\min
$$

where, $\max , \min$ is the feature range. Further, we have smoothened the signal so that we can capture only the sharpest of variations and eliminate the unwanted disturbances from the signal. We especially emphasized the baseline or the reference line of signal to be smooth. For this purposed, we applied a pre-processing technique that preserves the sharp rise and fall of the sensor data but eliminates the noise due to external environmental factors [42]. The objective of this algorithm was to minimize our cost function. According to the algorithm, the noisy signal is approximated as a combination of a piece-wise constant signal and noise, i.e.,

$$
y(n)=x(n)+w(n)
$$

where $x(n)$ is the piece-wise constant signal and $w(n)$ is the noisy part of our signal. Using the pre-processing technique, we can estimate $\mathrm{x}(\mathrm{n})$ by optimizing the following equation:

$\arg \min _{x}\left\{F(x)=\frac{1}{2} \sum_{n=0}^{N-1}|y(n)-x(n)|^{2}+\lambda \sum_{n=1}^{N-1}|x(n)-x(n-1)|\right\}$

The equation above can be written compactly by writing the $\mathrm{N}$-point signals as column vectors $\mathbf{x}$ and $\mathbf{y}$.

$$
\arg \min _{x}\left\{F(x)=\frac{1}{2}\|\mathbf{y}-\mathbf{x}\|\left\|_{2}^{2}+\lambda\right\| \mathbf{D x}||_{1}\right\}
$$

where $\mathbf{D}$ is an (N-1) X N matrix defined as, 


$$
\left[\begin{array}{ccccc}
-1 & 1 & & & \\
& -1 & 1 & & \\
& & \ddots & \ddots & \\
& & & -1 & 1
\end{array}\right]
$$

Dx will give the first-order difference of the signal $\mathbf{x}$, || $\mathbf{D} \mathbf{x}||_{1}$ gives the $1_{1}$ norm of the vector, and $\left.\|\mathbf{y}-\mathbf{x}\|\right|_{2}$ will give the $l_{2}$ norm of the vector.

We have used majorization-minimization (MM) technique [43] to approximate the minimum value of the cost function. The cost function can be represented as $\mathrm{F}(\mathbf{x})$, where, $\mathbf{x}$ is the vector representation of the N-point signal. The $\mathrm{MM}$ approach generates $\mathbf{x}_{\mathbf{k}}$ by minimizing $\mathrm{G}_{\mathrm{k}-1}(\mathbf{x})$, where, $\mathbf{x}_{\mathbf{k}}$ is the minimizer of $\mathrm{G}_{\mathrm{k}-1}(\mathbf{x})$, i.e., $\mathbf{x}_{\mathbf{k}}=\arg \min _{x} G_{k-1}(\mathbf{x})$. The obtained $\mathbf{x}_{\mathbf{k}}$ will converge to the minimum point of $F(\mathbf{x})$ if it is a convex function. The function, $\mathrm{G}_{\mathrm{k}}(\mathbf{x})$ is a majorizer that satisfies the following conditions:

(i) $\mathrm{G}_{\mathrm{k}}(\mathbf{x}) \geq \mathrm{F}$ (x) $\forall \mathbf{x}$, and

(ii) $\mathrm{G}_{\mathrm{k}}\left(\mathbf{x}_{\mathrm{k}}\right)=\mathrm{F}\left(\mathbf{x}_{\mathrm{k}}\right)$

For our cost function in eq. (5), we have chosen the majorizer as

$$
\frac{1}{2\left|t_{k}\right|} t^{2}+\frac{1}{2\left|t_{k}\right|} \geq|t|
$$

Replacing t with Dx, the equation becomes,

$$
\frac{1}{2} \mathbf{x}^{\mathbf{T}} \mathbf{D}^{\mathbf{T}}\left(\operatorname{diag}\left(\left|\mathbf{D} \mathbf{x}_{\mathbf{k}}\right|\right)\right)^{-1} \mathbf{D} \mathbf{x}+\frac{1}{2} \| \mathbf{D} \mathbf{x}_{\mathbf{k}}||_{1} \geq|| \mathbf{D} \mathbf{x}||_{1}
$$

To obtain a majorizer of our cost function as defined in eq. (7), we can add an additional term $\frac{1}{2}|| \mathbf{y}-\mathbf{x}||_{2}^{2}$ to both sides of eq. (9). We get,

$\frac{1}{2}|| \mathbf{y}-\mathbf{x}||_{2}^{2}+\lambda \frac{1}{2} \mathbf{x}^{\mathrm{T}} \mathbf{D}^{\mathrm{T}}\left(\operatorname{diag}\left(\left|\mathbf{D} \mathbf{x}_{\mathbf{k}}\right|\right)\right)^{-1} \mathbf{D x}+\lambda \frac{1}{2}|| \mathbf{D} \mathbf{x}_{\mathbf{k}}||_{1} \geq \frac{1}{2}|| \mathbf{y}-\left.\mathbf{x}\right|_{2} ^{2}+\lambda|| \mathbf{D x}||_{1}$

From this, we can obtain a sequence $\mathbf{x}_{\mathbf{k}}$ by minimizing the above majorizer function. A solution for the same has been obtained as follows:

$$
\mathbf{x}_{\mathbf{k}+\mathbf{1}}=\left(\mathbf{I}+\lambda \mathbf{D}^{\mathbf{T}}\left(\operatorname{diag}\left(\left|\mathbf{D} \mathbf{x}_{\mathbf{k}}\right|\right)\right) \mathbf{D}\right)^{-1} \mathbf{y}
$$

In order to avoid any inconsistent matrices, we can rewrite the equation using the matrix inverse lemma. The equation can then be written as,

$$
\mathbf{x}_{\mathbf{k}+\mathbf{1}}=\mathbf{y}-\mathbf{D}^{\mathbf{T}}\left(\frac{1}{\lambda} \operatorname{diag}\left(\left|\mathbf{D} \mathbf{x}_{\mathbf{k}}\right|\right)+\mathbf{D D}^{\mathbf{T}}\right)^{-1} \mathbf{D} \mathbf{y}
$$

The solution to this equation can be easily found by solving for the system of linear equations. The solution is not very computationally intensive because $\mathbf{D D}^{\mathbf{T}}$ is a tridiagonal matrix that can be solved very quickly. The above method will give us a smoother signal.

The next method that we applied to the signal is to bring the majority of reference line values to zero. This will help us achieve a uniform reference level for all signals. Some of the signals obtained from the sensors have drifted away from the reference value, as can be seen in fig. 6. For accurate measurement of LWD values, it is imperative that the reference values remain consistent throughout the duration of the signal. The baseline correction applied to the signal is a combination of smoother and asymmetrically weighted deviations of smoothened signal from the raw signal [44].

The baseline values should fit well to the actual signal. To ensure that, the following equation is optimized:

$$
\begin{gathered}
F=\sum_{n=0}^{N-1} w(n)[y(n)-x(n)]^{2}+\lambda \sum_{n=1}^{N-1}\{(y(n)-x(n))-(y(n-1)-x(n-1))\}^{2} \\
+\lambda \sum_{n=2}^{N-1}\{(x(n)-x(n-1))-(x(n-1)-x(n-2))\}
\end{gathered}
$$

Here, $w(n)$ is the weight of fitness. The above equation is then minimized and the solution can be written in vector form as:

$$
\mathbf{x}=\left\{\mathbf{W}^{\mathrm{T}} \mathbf{W}+\lambda\left(\mathbf{D}^{\mathbf{T}} \mathbf{D}+\left(\mathbf{D D}^{\mathrm{T}}\right)^{2}\right)\right\}^{-1}\left\{\mathbf{W}^{\mathrm{T}} \mathbf{W}+\lambda \mathbf{D}^{\mathbf{T}} \mathbf{D}\right\} \mathbf{y}
$$

where $\mathbf{W}=\operatorname{diag}(w(n))$. The signal thus obtained is smoothened and baseline corrected as can be seen in fig. 7 .

In the next section, we have mentioned the methodology used for calculating LWD values. It mentions how we have identified the starting and ending points of the events and calculated the duration for each of them.

\section{B. Feature Extraction}

Fig. 7 represents a wholly processed signal. The baseline or reference line of the signal has very little variation. As seen in the raw signal, the small disturbances are represented in fig. 6 are almost eliminated. The reference values have also been brought down to 0 by doing the baseline correction. Most of the reference values are now on a consistent level which was not necessarily the raw signal. These modifications will drastically reduce the errors in the computation of the LWD values.

The events, as mentioned before, could be due to dew, rain, or irrigation. To find the LWD values, we need to identify the points where the signal becomes greater than the reference value. We have set the threshold as $5 \%$, 
variations beyond which are considered to be due to either of the phenomena mentioned above. The event's starting point is considered to be the one where the value of the signal becomes greater than the threshold level right after it was lower than the threshold. Similarly, the endpoint is when the value of the signal is greater than the threshold right before it becomes less than the same. We have captured these two points for all the events that have occurred in the given signal. We collected data over a month's time period during which our sensors could capture 17 events for our study.

Fig. 8 shows the marked points for identifying the events. The points marked with an orange marker are the starting points of the events. The points marked with a green marker represent the end of the events. Then, the timestamps of these points have been consolidated together. The duration has been easily calculated by subtracting the start time from the stop time and is expressed in hours. Fig.9 shows the pictorial representation of the LWD values from all the sensors. From the graph, it can be observed that most of the LWD values calculated are consistent across all four sensors. A few of them have minor differences of 30 minutes to 1 hour, which are acceptable for integrated plant disease management applications. This difference is attributed to the data sampling rate during in-situ measurements, which is about 30 minutes.

\section{Results AND Discussion}

In this work, we explored the potential use of the electronic leaf wetness sensor fabricated on the flexible substrates. Considering the issues associated with the operational exposure of the reported LWS, where the weight of the sensor and contact resistance are few concerns. Thus, to mitigate the above issues associated with the available LWS, we have fabricated the LWS on the flexible substrates in this work. The weight of the fabricated sensor is less than $1 \mathrm{gm}$, and thus, it will not bend the leaves during the field deployment. Fabricated LWS is on flexible substrates; thus, it offers better contact resistance between the leaf surface and the sensor. This illustrates the novelty of the proposed work when compared with [23].

We have developed the IoT-enabled system for the fabricated sensor and deployed this system on the Ocimum tenuiflorum (Tulsi) medical plant. The dimension of the fabricated flexible LWS is designed considering the dimension of Tulsi plant leaves, which makes this sensor novel compared with the [21]. To analyze and understand the performance of the fabricated flexible LWS in terms of accuracy, we carried out controlled field experiments and benchmarked the measured LWD using the fabricated flexible LWS and commercially available LWS. For this purpose, the fabricated LWS and system are tested under in-situ conditions for about one month and commercial LWS Phytos 31, where we collected about 1600 data points. Subsequently, the data preprocessing is done on the raw sensor data and the calculated LWD for both the sensor viz. fabricated and commercial LWS. The field measurement results indicate that the discrepancy in the measured LWD using the fabricated flexible LWS and Phytos 31 is about 30 minutes to 1 hour. Thus, fabricated LWS can be considered as one of the potential candidates considering the in -situ measurements.
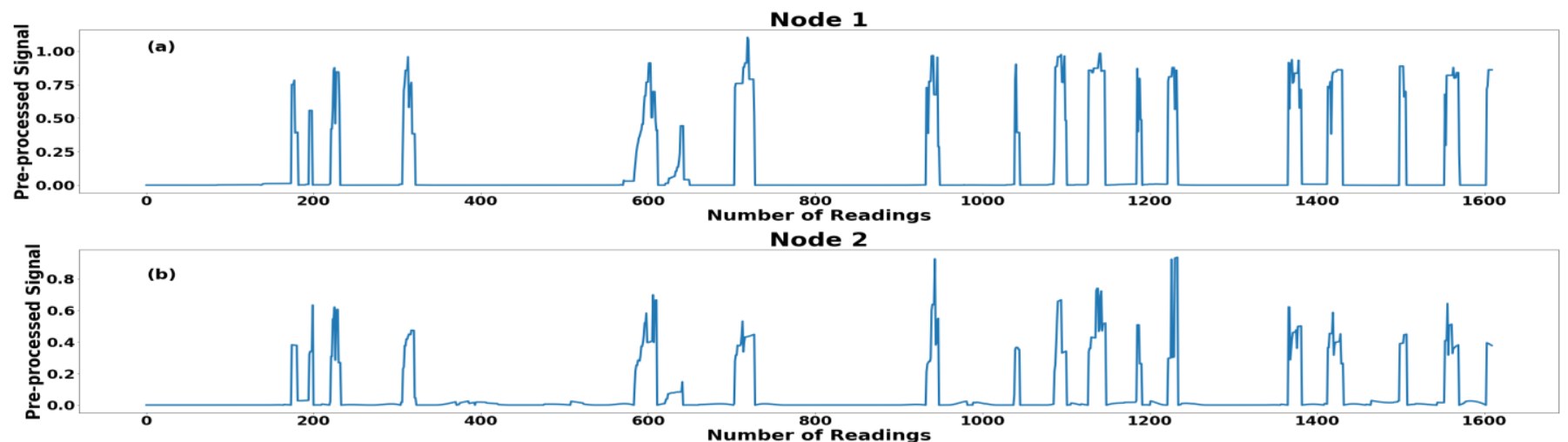

Node 3
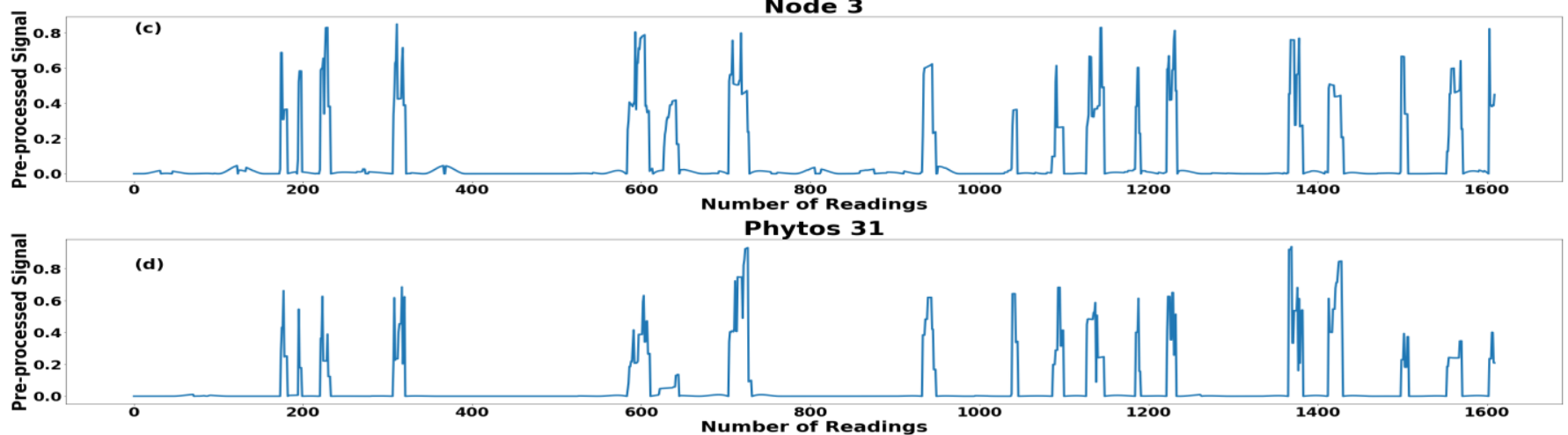

Fig. 7 Raw signal from the four sensors with normalized values 

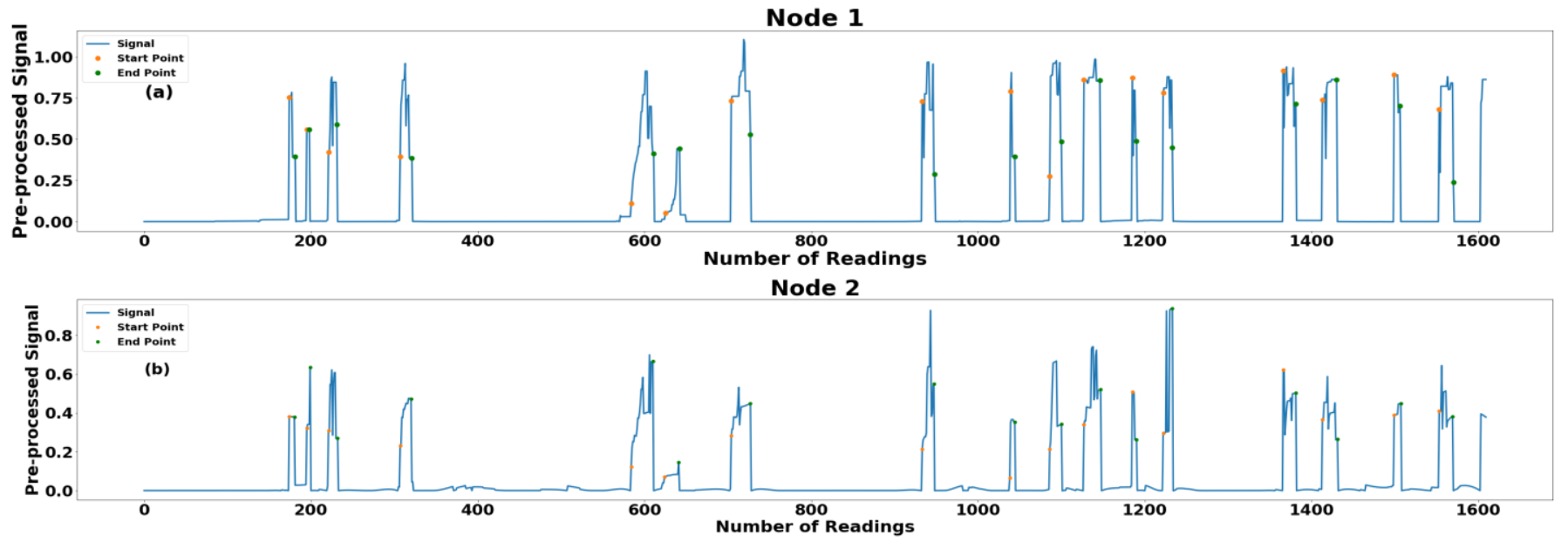

Node 3

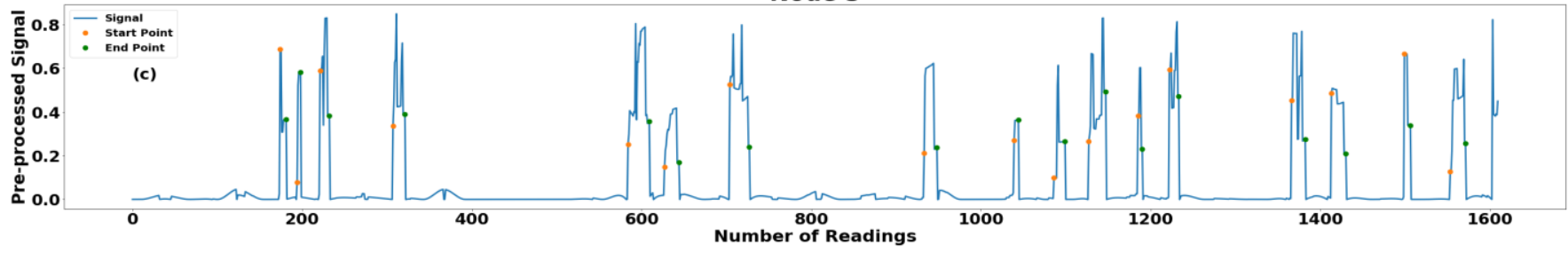

Phytos 31

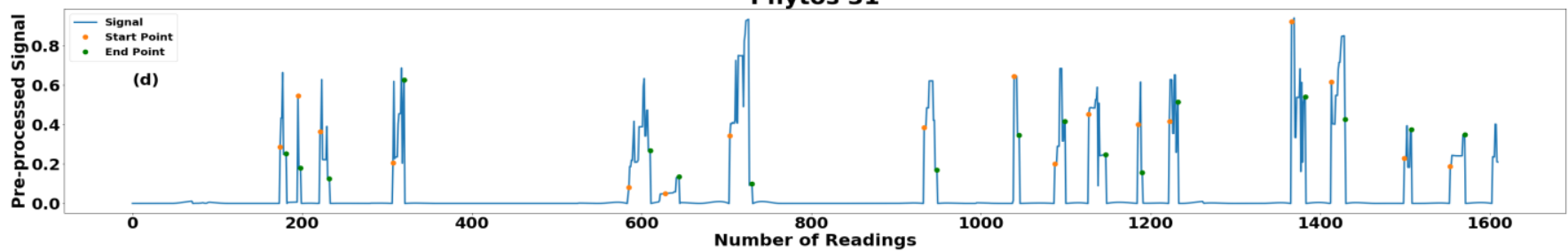

Fig. 8 Pre-processed signal with smoothened and corrected baseline values and starting and ending points of all 17 events observed

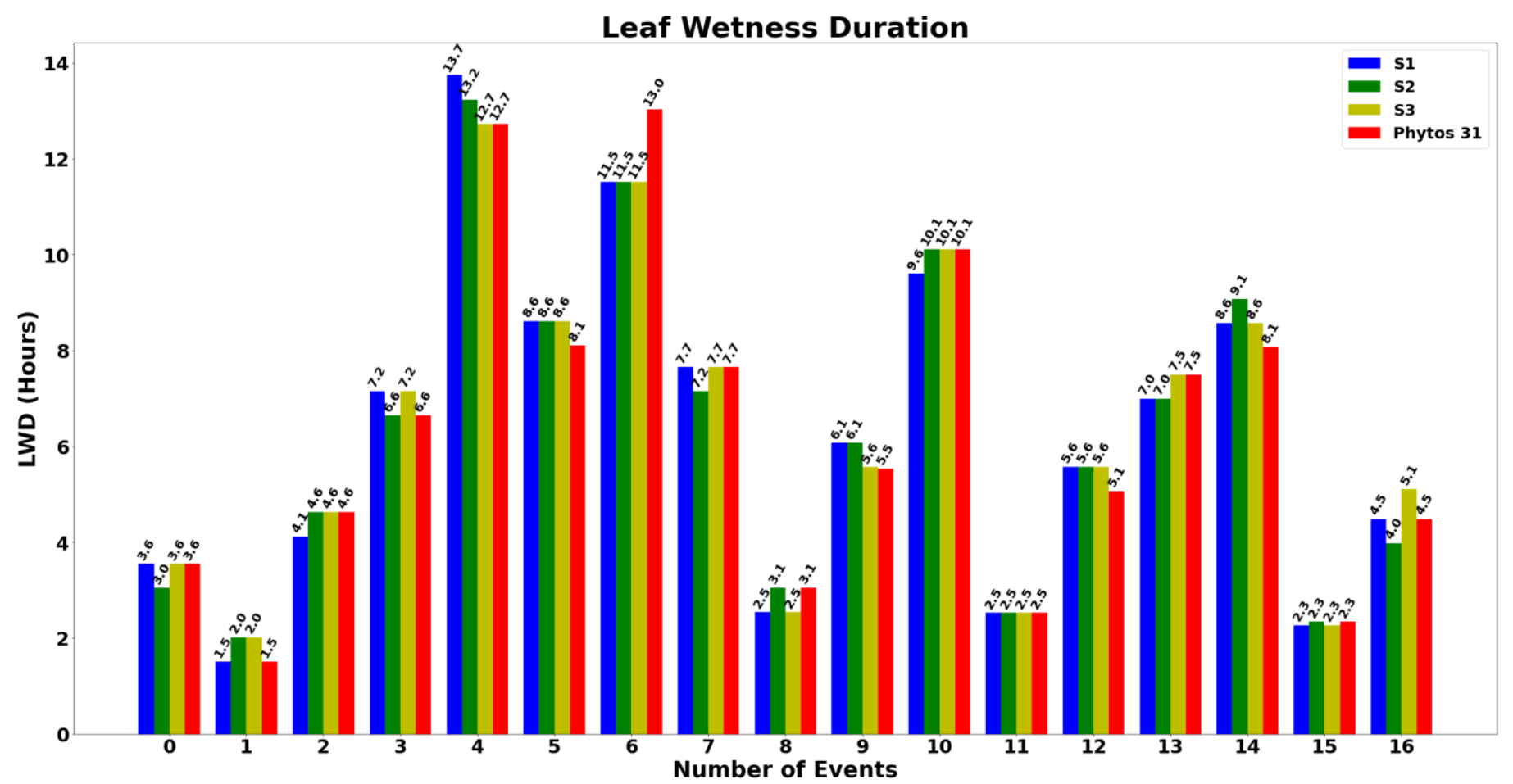

Fig. 9 Comparison of LWD values from all the four sensors using bar graph. 


\section{CONCLUSIONS}

Plant disease monitoring and early prediction is very important to reduce the crop yield. To identify the probability of plant diseases, information on LWD is very important, and to measure it, LWS is widely used. We have fabricated the LWS on the flexible substrates and developed the IoTenabled system to record the sensor data in this work. Fabricated flexible LWS performance is examined under both lab and the field conditions. The lab measurements indicate that the fabricated flexible LWS offers a response of about $36000 \%$ when the sensing area is completely wet w.r.t air. Further, it is observed that the response is about 10 seconds and hysteresis of about $\pm 4 \%$ for the fabricated LWS. Interestingly, the LWS sensor capacitance changes only by $6 \%$ when temperature varies from $20^{\circ} \mathrm{C}$ to $65^{\circ} \mathrm{C}$., making this one of the potential candidates for the field measurements. Furthermore, during the field testing of the fabricated flexible LWS, we have benchmarked the measured LWD with commercial expensive LWS Phytos 31. Field measurement results indicate that the discrepancy in the LWD is about 30 minutes to 1 hour, which is acceptable for integrated plant disease management. Thus, LWS fabricated on flexible substrate stands out to be one of the potential candidates for the in-situ measurements considering both the price and accuracy. As a future scope, we will collect data for one crop cycle and make the early prediction of the plant disease using the fabricated LWS.

\section{ACKNOWLEDGMENT}

The authors are thankful to would like to thank the Department of Science and Technology - Science and Engineering Research Board (DST-SERB) for financial assistance received through a start-up research grant (SRG) FILE NO. SRG/2019/000895. We would like to extend our thanks to the staff of Nanofabrication Core Lab, King Abdullah University of Science and Technology (KAUST), Saudi Arabia for providing assistance in the project.

\section{REFERENCES}

[1] A. Sajeena, J. John, B. Sudha, A. V. Meera, and S. R. Karthika, "Significance of Botanicals for the Management of Plant Diseases", In Plant Health Under Biotic Stress, pp. 231-243. Singapore, 2019.

[2] M. G. Lawrence, "The relationship between relative humidity and the dew point temperature in most air: a simple conversion and applications", Bull. Am. Meteorol. Soc. 86, pp.225-233, 2005.

[3] L. L. Granke, and M. K. Hausbeck, "Effects of temperature, humidity, and wounding on development of Phytophthora rot of cucumber fruit." Plant disease. 94 (12), pp. 1417-1424, 2010.

[4] M. Kumar, A. Kumar, and V. S. Palaparthy, "Soil Sensors Based Prediction System for Plant Diseases using Exploratory Data Analysis and Machine Learning." IEEE Sensors Journal (2020).
[5] L. Huber and T. J. Gillespie. "Modeling leaf wetness in relation to plant disease epidemiology." Annual review of phytopathology, 30 (1), pp. 553-577, 1992.

[6] Y. Q. Wei, B. J. Bailey, and B. C. Stenning. "A wetness sensor for detecting condensation on tomato plants in greenhouses." Journal of agricultural engineering research, 61 (3), pp. 197-204, 1995.

[7] T. S. Gillespie, T.S. and G. E. Kidd, "Sensing duration of leaf moisture retention using electrical impedance grids". Can. J. Plant Sci., 58, pp. 179-187, 1978.

[8] H. Haickel, "New developments of an electrical method for direct measurement of the wetness-duration on plants", Agric. Meteorol. 22, pp.113-119, 1980.

[9] R.R. Getz, "Report on the measurement of leaf wetness", WMO (Commission for Instruments and Methods of Observation), Rome, 1991.

[10] T. J. Gillespie, R. X. Duan, "A comparison of cylindrical and flat plate sensors for surface wetness duration”, Agric. For. Meteorol. 40, pp. 61-70, 1987.

[11] T. J. Gillespie, B. Srivastava, R. E. Pitblado, "Using operational weather data to schedule fungicide sprays on tomatoes in Southern Ontario", Can. J. Appl. Meteorol. 32, pp. 567-573, 1993.

[12] M.L. Gleason, S.E. Taylor, T.M. Loughin, K.J. Joehler, "Development and validation of an empirical model to estimate the duration of dew periods". Plant Dis. 78, pp. 1011-1016, 1994.

[13] K. S. Kim, S.E. Taylor, M.L. Gleason, K.J. Koehler, "Model to enhance site-specific estimation of leaf wetness duration”, Plant Dis. 86, pp. 179-185, 2002.

[14] P.S. Rao, T.J. Gillespie, A.W. Schaafsma, "Estimating wetness duration on maize ears from meteorological observations", Can. J. Soil Sci. 78, pp. 149-154, 1998.

[15] P. C. Sentelhas, J.E.B.A. Monteiro, T.J. Gillespie, "Electronic leaf wetness duration sensor: why it should be painted". Int. J. Biometeorol. 48, pp. 202-205, 2004.

[16] Y. Q. Wei, B. J. Bailey, B.C. Stenning, "A wetness sensor for detecting condensation on tomato plants in greenhouses", J. Agric. Eng. Res. 61, 197-204, 1995.

[17] A. Weiss, D. L. Lukens, "Electronic circuit for detecting leaf wetness and comparison of two sensors", Plant Dis. 65, pp. 41-43, 1981.

[18] A. Weiss, A.F. Hagen, "Further experiments on the measurement of leaf wetness". Agric. Meteorol. 29, pp. 207-212, 1983.

[19] A. Weiss, A.F. Lukens, J. R. Steadman, "A sensor for the direct measurement of leaf wetness: construction techniques and testing under controlled conditions", Agric. For. Meteorol. 43, 241-249, 1988.

[20] Z. Gao, W. Shi, X. Wang, and Y. Wang. "Non-rainfall water contributions to dryland jujube plantation evapotranspiration in the Hilly Loess Region of China." Journal of Hydrology 583, pp. 124604, 2020.

[21] PHYTOS 31, User Manual, METER Group, Inc. USA, Link:

http://library.metergroup.com/Manuals/20434_PHYTOS31

_Manual_Web.pdf (Date of visit: 17th November 2020) 
[22] S. Bregaglio, D. Marcello, C. Roberto, A. Marco, and S. Orlandini. "Multi metric evaluation of leaf wetness models for large-area application of plant disease models." Agricultural and Forest Meteorology 151, 9, pp, 1163-1172, 2011.

[23] G. Hornero, J. E. Gaitán-Pitre, E. S. Finetti, O. Casas, and R. Pallas-Areny. "A novel low-cost smart leaf wetness sensor." Computers and electronics in agriculture 143, pp. 286-292, 2017.

[24] D. R. Davis, and J. E. Hughes. "A new approach to recording the wetting parameter by the use of electrical resistance sensors." Plant Disease Reporter 54 (6), pp. 474 479, 1970.

[25] A. C. Madeira, K. S. Kim, S. E. Taylor, M. L. Gleason, "A simple cloud-based energy balance model to estimate dew”, Agric For Meteorol 111, pp. 55-63, 2002.

[26] RAC Miranda, T. D. Davie, S. E. Cornell SE, "A laboratory assessment of wetness sensors for leaf, leaf, fruit and trunk surfaces", Agric For Meteorol 102:263-274, 2000.

[27] P. C. Sentelhas, T. J. Gillespie, M. L. Gleason, J. Eduardo BA Monteiro, and S. T. Helland. "Operational exposure of leaf wetness sensors." Agricultural and Forest Meteorology 126, no. 1-2, pp.59-72, 2004.

[28] P. Aravind, M. Gurav, A. Mehta, R. Shelar, J. John, V. S. Palaparthy, K. K. Singh, S. Sarik, and M. S. Baghini. "A wireless multi-sensor system for soil moisture measurement." In 2015 IEEE SENSORS, pp. 1-4. IEEE, 2015.

[29] H. Bi, K. Yin, X. Xie, J. Ji, S. Wan, L. Sun, M. Terrones, and M. S. Dresselhaus. "Ultrahigh humidity sensitivity of graphene oxide." Scientific reports 3, no. 1, pp. 1-7, 2013.

[30] V. S. Palaparthy, H. Kalita, S. G. Surya, M. S. Baghini, and M. Aslam. "Graphene oxide based soil moisture microsensor for in situ agriculture applications." Sensors and Actuators B: Chemical 273, pp. 1660-1669, 2018.

[31] I. Mouhamad, J. Claudel, D. Kourtiche, and M. Nadi. "Geometric parameters optimization of planar interdigitated electrodes for bioimpedance spectroscopy." Journal of Electrical Bioimpedance 4, 1, pp.13-22, 2013.

[32] H. Kalita, V. S. Palaparthy, M. S. Baghini, and M. Aslam. "Graphene quantum dot soil moisture sensor." Sensors and Actuators B: Chemical 233, pp. 582-590, 2016. [33] S. G. Surya, S. Yuvaraja, E. Varrla, M. S. Baghini, V. S. Palaparthy, and K. N. Salama. "An in-field integrated capacitive sensor for rapid detection and quantification of soil moisture." Elsevier Sensors and Actuators B: Chemical 321, Pp 128542, 2020.

[34] HIH-5030/5031 (Datasheet), Low Voltage Humidity Sensors, Honeywell Sensing and Control, Link: https://sensing.honeywell.com/honeywell-sensing-hih50305031-series-product-sheet-009050-2-en.pdf (Date of visit: 17th November 2020)

[35] MCP9701 (Datasheet), Low-Power Linear Active Thermistor ICs, 2005-2016 Microchip Technology Inc.

[36] ATmega328P [DATASHEET], 7810D-AVR-01/15, Link:

https://ww1.microchip.com/downloads/en/DeviceDoc/Atme 1-7810-Automotive-Microcontrollers-
ATmega328P_Datasheet.pdf (Date of visit: 17th November 2020)

[37] ESP-01 WiFi Module, Version1.0, Link: http://www.microchip.ua/wireless/esp01.pdf (Date of visit: 17th November 2020)

[38] LCR METER IM3536, HIOKI, Jan. 12, 2018 Link: https://www.hioki.com/file/cmw/hdCatalog/4334/pdf/?actio $\mathrm{n}=$ browser\&log=1\&lang=en (Date of visit: 17 th November 2020).

[39] V. S. Palaparthy et al., "E-Nose: Multichannel Analog Signal Conditioning Circuit With Pattern Recognition for Explosive Sensing," in IEEE Sensors Journal, vol. 20, no. 3, pp. 1373-1382, 1 Feb.1, 2020

[40] V. S. Palaparthy et al., "Hybrid Pattern Recognition for Rapid Explosive Sensing With Comprehensive Analysis," in IEEE Sensors Journal, vol. 21, no. 6, pp. 8011-8019, 15 March15, 2021

[41] Pedregosa, Fabian, Gaël Varoquaux, Alexandre Gramfort, Vincent Michel, Bertrand Thirion, Olivier Grisel, Mathieu Blondel et al. "Scikit-learn: Machine learning in Python." the Journal of machine Learning research 12, pp. 2825-2830, 2011.

[42] Figueiredo, Mario AT, J. Bioucas Dias, João Pedro Oliveira, and Robert D. Nowak. "On total variation denoising: A new majorization-minimization algorithm and an experimental comparisonwith wavalet denoising." 2006 International Conference on Image Processing, pp. 26332636. IEEE, 2006.

[43] Figueiredo, Mário AT, José M. Bioucas-Dias, and Robert D. Nowak. "Majorization-minimization algorithms for wavelet-based image restoration." IEEE Transactions on Image processing 16, no. 12 , pp. 2980-2991, 2007.

[44] He, Shixuan, Wei Zhang, Lijuan Liu, Yu Huang, Jiming $\mathrm{He}$, Wanyi Xie, Peng $\mathrm{Wu}$, and Chunlei Du. "Baseline correction for Raman spectra using an improved asymmetric least squares method." Analytical Methods 6, no. 12, pp. 4402-4407, 2014.

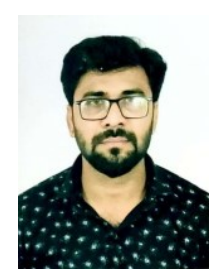

Kamlesh S. Patle is currently working as a junior research fellow (JRF) at DA-IICT Gandhinagar, Gujarat. India, He received his M.Sc. degree from Nagpur University, Nagpur, India. $\mathrm{He}$ has qualified UGC-NET and SET in 2013. Prior to joining as a JRF, he was working as an embedded engineer in StepOne Technologies Pvt. Ltd for four years, where he was actively involved the embedded hardware development. He has 2 publications on his ongoing work. His area of interested include the sensor and system development considering in-situ aspects and affordability.

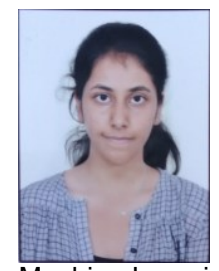

Riya Saini completed her Bachelor's in Engineering from RV College of Engineering, Bengaluru in the year, 2020. She qualified the GATE examination for Instrumentation Engineering in the same year. Currently, she is working as a Junior Research Fellow (JRF) at DA-IICT Gandhinagar, Gujarat, India. Her interest is to pursue research. in the, which includes Machine Learning and Deep Learning. 


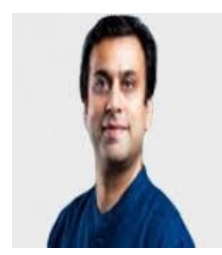

Ahlad Kumar has joined DA-IICT in July 2019. He has served as the postdoctoral fellow in Concordia University and won the prestigious Horizon Postdoctoral Fellowship at Concordia University, Montreal, Canada in 2017. Dr. Kumar holds a Ph.D. from the University of Malaya, Malaysia in 2016. He has received the gold medal for his performance during M.Tech. (VLSI) from ABV-Indian Institute of Information Technology and Management, Gwalior in 2007. He received the B.Tech. degree in Electronics and Communication Engineering from Jamia Millia Islamia in 2005.

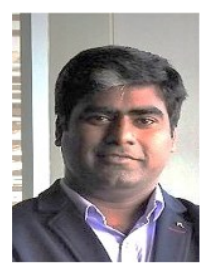

Sandeep G Surya is currently a Postdoctoral Research Fellow at King Abdullah University of Science and Technology (KAUST), Kingdom of Saudi Arabia. He received his Ph.D. degree from IIT Bombay and B.Tech. degree from JNTU, Hyderabad. After graduation, he worked as Research Associate at Microsystems Technology Research Unit, CMM, Fondazione Bruno Kessler (FBK), Trento, Italy (2017). He has 45 research publications and 5 patents related to his research. He was a member of different teams working on explosive detectors and Telemedicine. His work won GANDHIAN YOUNG TECHNOLOGICAL INNOVATION AWARD at IIM-A 2012, the Fellowship at ISED 2011, the best novelty prize for a humanoid robot at IIIT-H and the P.P CHHABRIA AWARD OF EXCELLENCE in intelligent systems and robotics for the year 2009 at IIIT, Pune.

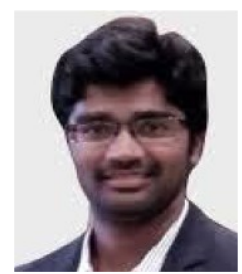

Vinay S Palaparthy is currently working as an Assistant professor in the Department of Information and Communication Technology, DAIICT, Gandhinagar, India. He received $\mathrm{Ph} . \mathrm{D}$. from the Indian Institute of Technology Bombay and an M.Sc degree with the first rank from Nagpur University, Nagpur, India. He was awarded INSPIRE Fellowship for pursuing Ph.D. degree from the Department of Science and Technology (DST). He is currently working in the field of MEMS/NEMS sensor design, 2D Nanomaterials and embedded system design primarily focusing on the in-situ agriculture applications. He has 10 international journals 8 conference papers and 1 Indian patent awarded on his ongoing work. He has one best paper award in an international journal. $\mathrm{He}$ is a co-recipient of Millennium Alliance award for the start-up name Proximal Soilsens Technologies Pvt. Ltd, where he is one of co-founder.

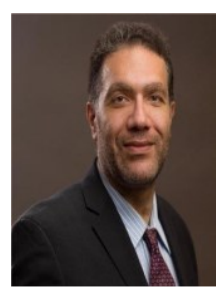

Khaled N. Salama received the B.S. degree from the Department Electronics and Communications, Cairo University, Cairo, Egypt, in 1997, and the M.S. and Ph.D. degrees from the Department of Electrical Engineering, Stanford University, Stanford, CA, USA, in 2000 and 2005, respectively. He was an Assistant Professor at Rensselaer Polytechnic Institute, NY, USA, between 2005 and 2009. He joined King Abdullah University of Science and Technology (KAUST) in January 2009, where he is now a professor, and was the founding Program Chair until August 2011. $\mathrm{He}$ is the director of the sensors initiative a consortium of 9 international universities. His work on CMOS sensors for molecular detection has been funded by the National Institutes of Health (NIH) and the Defense Advanced Research Projects Agency (DARPA), awarded the Stanford-Berkeley Innovators Challenge Award in biological sciences and was acquired by Illumina Inc. He is the author of 300 papers and 20 US patents on lowpower mixed signal circuits for intelligent fully integrated sensors and neuromorphic circuits using memristor devices. 